\title{
Manejo mixto: Anestesia general balanceada y bloqueo subaracnoideo en cirugía bariátrica con paciente pediátrico
}

\author{
Almonte De León A. ${ }^{1,2,3}$ \\ 1 Hospital Santa Barbara del Rieti, Durango, México. \\ 2 Hospital General 450, Servicios de Salud, Durango, México. \\ 3 Instituto Mexicano del Seguro Social, Durango, México. \\ Introducción: La obesidad infantil es un problema de salud pública grave del siglo XXI. Es mundial y está afectando \\ progresivamente a países de bajos y medianos ingresos, sobre todo en el medio urbano. 1) La prevalencia ha aumen- \\ tado a ritmo alarmante. Paralelamente al incremento de prevalencia del adulto, la obesidad del adolescente está \\ aumentando; 2) Los procedimientos bariátricos establecen una forma de reducción de peso; 3) El niño obeso presenta \\ aumento de comorbilidades, además de dificultad en el manejo perioperatorio e incremento en las complicaciones \\ perioperatorias; 4) El procedimiento laparoscópico reduce tiempo hospitalario y dolor; 5) Aun permanecen muchas \\ dudas sobre seguridad y eficacia de cirugía bariátrica en niños y adolescentes.
}

Objetivo general: Describir manejo mediante bloqueo neuroaxial en combinación con anestesia general balanceada para paciente pediátrico en cirugía bariátrica.

Caso clínico: Femenino 13 años de edad con diagnósticos: obesidad exógena + resistencia a la acción de la insulina + hiperlordosis dorsolumbar + talo valgo y genu varo. Peso: $103 \mathrm{~kg}$. Talla 1,63, IMC 39,7 kg/m2sc. Procedimiento quirúrgico: manga gástrica. Valoración por pediatría, endocrinología, psiquiatría y nutrición sin contraindicaciones para procedimiento. Bajo bloqueo neuroaxial con bupivacaína 12,5 mg + morfina $100 \mathrm{mcgr}$. Anestesia General Balanceada con midazolam $2 \mathrm{mg}$, fentanilo $200 \mathrm{mcgr}$ propofol $200 \mathrm{mg}$, atracurio $25 \mathrm{mg}$. Tubo Endotraqueal \#7. Mantenimiento sevofluorane volumen promedio $2 \%$. Transanestésico hemodinámicamente estable. Tasa fentanilo 2,57. Emersión por lisis metabólica, extubación sin incidentes. Aldrete 8 EVA 0 Bromage 2.

Discusión: Hay escasez de información específica de la farmacocinética y farmacodinamia de muchos fármacos frecuentes en niños obesos y las conclusiones se han extraído en gran medida de los trabajos realizados en adultos. La literatura disponible frecuentemente contiene resultados conflictivos para el mismo agente, dependiendo de la edad y el grado de la obesidad estudiada. El manejo con anestesia regional no está descrito para uso común según la literatura, sin embargo, disminuye los requerimientos anestésicos.

Conclusiones: El manejo del paciente pediátrico sometido a cirugía bariátrica con anestesia general balanceada en combinación con anestesia neuroaxial disminuye los requerimientos de anestésicos intravenosos y agentes inhalados y provee una adecuada analgesia postoperatoria.

https://doi.org/10.25237/congresoclasa2019.64 